\title{
GLOBALIZACIÓN, MIGRACIÓN Y ALGUNOS TRABAJOS QUE NO TIENEN FIN
}

ELAINE LEVINE*

\section{Resumen}

Este trabajo analiza la creciente complementariedad y la integración de facto entre el mercado laboral mexicano y el estadounidense. Se parte de los cambios en la estructura del empleo en Estados Unidos y la creciente polarización y segmentación del mercado laboral de aquel país para explicar cómo es que, mientras millones de hombres estadounidenses han visto truncada su vida laboral por la reestructuración y relocalización industrial, millones de migrantes mexicanos encuentran empleos, predominantemente en el creciente sector de servicios, algunos rubros de la manufactura ligera y en el menguante (desde el punto de vista laboral) sector agrícola - aun cuando son empleos no muy deseables y muy mal pagados, según los parámetros estadounidenses- que les proporcionan ingresos 10 o 15 veces superiores a los que podrían lograr en México, en caso de conseguir trabajo. Cabe señalar que la crisis iniciada en 2007 no ha provocado el retorno masivo de migrantes mexicanos, solamente ha mitigado el flujo de estos hacia Estados Unidos en los últimos años.

Palabras clave: migración mexicana, segmentación del mercado laboral, nichos laborales para inmigrantes, estratificación socioeconómica, reestructuración económica.

* Profesora y tutora del Posgrado en Ciencias Políticas y Sociales de la UnAM. Dirección electrónica: elaine@servidor.unam.mx. 


\begin{abstract}
This article analyzes the increasing complementarity between the Mexican and us labor markets and the de facto integration taking place. Changes in the employment structure in the US and the increasing polarization and segmentation of the labor market there help explain how millions of Mexican immigrants find jobs north of the border while millions of men in the Us have seen their working lives cut short, because of industrial restructuring and relocation. Mexican immigrants find jobs mainly in the growing service sector, some branches of light manufacturing, and the waning (from an employment perspective) agricultural sector -even though they may be undesirable and low paying jobs by US standards- that provide them with incomes ten or even fifteen times higher than what they could earn in Mexico if they were employed. Furthermore, the crisis that began at the end of 2007 has not caused a massive return to Mexico it has merely diminished the incoming flow of new immigrants.
\end{abstract}

Keywords: Mexican immigration, labor market segmentation, immigrant labor niches, socio-economic stratification, economic restructuring. 


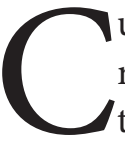
uando, en 1995, Jeremy Rifkin publicó su libro El fin del trabajo, recibió elogios que catalogaban su trabajo como visionario; pero también críticas que lo tachaban de absurdo. Para los más de 4 millones de hombres de entre 30 y 55 años de edad que dejaron de buscar empleo en Estados Unidos en el primer lustro del siglo xxI, resultó premonitor (Uchitelle y Leonhardt, 2006): solamente entre 2000 y 2005 desaparecieron 3 millones de empleos en el sector manufacturero. Entre 2005 y 2010, en el contexto de la recesión más severa desde la crisis de los años treinta, desaparecieron otros 2.6 millones de empleos manufactureros (USDOL, 2011). Sin embargo, para los millones de migrantes mexicanos que se han ido al norte en las últimas tres décadas, el país vecino ofrece posibilidades de empleo e ingresos que no encuentran en sus lugares de origen. La globalización y las innovaciones tecnológicas e informáticas han trastornado y transformado los mercados laborales en muchos países. En consecuencia, ha cambiado radicalmente la estructura del empleo, tanto del lado de la demanda como de la oferta de mano de obra. Con la proliferación del knowledge based work (trabajo basado en el conocimiento), puede ser que el concepto mismo de trabajo se esté modificando.

En este estudio analizaremos la creciente complementariedad y la integración de facto entre el mercado laboral mexicano y el estadounidense; partiremos de los cambios en la estructura del empleo en Estados Unidos, y la creciente polarización y segmentación del mercado laboral de aquel país para explicar cómo es que, mientras millones de hombres estadounidenses han visto truncada su vida laboral, por la reestructuración y relocalización industrial, millones de migrantes mexicanos encuentran empleos, predominantemente en el creciente sector de servicios, algunos rubros de la manufactura ligera y en el menguante (desde el punto de vista laboral) sector agrícola - aun cuando son empleos no muy deseables y muy mal pagados, según los parámetros estadounidenses- que les proporcionan ingresos $10 \mathrm{o} 15$ veces superiores a los que podrían lograr en México, en caso de conseguir trabajo. Cabe señalar que la crisis iniciada en 2007 no ha provocado el retorno masivo de migrantes mexicanos; solamente ha mitigado el flujo de estos hacia Estados Unidos en los últimos años (Passel y Cohn, 2011). Además, el número de indocumentados en aquel país ha disminuido, principalmente, no por salidas voluntarias sino por el incremento en las deportaciones. 


\section{LA CAMBIANTE ESTRUCTURA OCUPACIONAL EN ESTADOS UNIDOS}

A lo largo de la posguerra, el crecimiento económico y demográfico de Estados Unidos ha sido acompañado por un aumento más o menos regular, aun cuando lento en términos porcentuales, en la población económicamente activa (PEA), lo cual ha derivado en un incremento importante en el número de personas empleadas. El porcentaje de la PEA con respecto al total pasó del $59.2 \%$ en 1950 a $65.7 \%$ en 2010 ; mientras que, en términos absolutos, la fuerza laboral creció $155 \%$ (USDOL, 1984 y 2011). Sin embargo, importantes cambios económicos y sociales - revolución científico-tecnológica y revolución informática, transnacionalización de las empresas, movimiento por los derechos civiles de la población afro-americana, movimiento feminista, globalización, reestructuración industrial y políticas económicas neoliberales, por mencionar algunos- han modificado sustancialmente la estructura del empleo y las características de la PEA.

En primer lugar, el empleo agrícola ha disminuido marcadamente tanto en términos absolutos como relativos: los 9.5 millones de personas ocupados en actividades agrícolas en 1940 constituían el 20\% de la PEA; pero los 2.2 millones ocupados en ese sector en 2010 representaban apenas el 1.4\%. No obstante lo anterior, Estados Unidos es uno de los productores y exportadores más importantes de productos agrícolas a nivel mundial.

Aun cuando Robert Reich (1992:85-86) plantea que la distinción tradicional entre bienes y servicios carece de sentido en nuestros días, no deja de llamar la atención que un porcentaje creciente de la PEA se dedica a la producción o provisión de servicios (62.1\% en 1955 frente a $86.2 \%$ en 2010 ) y una proporción decreciente (37.9\% en 1955 comparado con $13.7 \%$ en 2010) se ocupa en la producción de bienes (ver tabla 1 , USDOL, 2006 y 2011). De todas formas, y en parte gracias a un creciente déficit comercial, la gama de productos disponibles para los consumidores estadounidenses es mayor que nunca. 
TABLA 1

Distribución Sectorial del Empleo en Estados Unidos

\begin{tabular}{|c|c|c|c|c|c|c|c|c|}
\hline EMPLEADOS NO AGRICOLAS POR SECTOR INDUSTRIAL (MILES) & 1955 & $\%$ & 1979 & $\%$ & 2005 & $\%$ & 2010 & $\%$ \\
\hline Total (no agrícola) & 50,744 & $100 \%$ & 89,932 & $100 \%$ & 133,631 & $100 \%$ & 130,262 & $100 \%$ \\
\hline Total privado (no agrícola) & 43,722 & $86.2 \%$ & 73,864 & $82.1 \%$ & 111,836 & $83.7 \%$ & 107,791 & $82.7 \%$ \\
\hline \multicolumn{9}{|l|}{ PRODUCCIÓN DE BIENES } \\
\hline Total & 19,234 & $37.9 \%$ & 24,997 & $27.8 \%$ & 21,141 & $15.8 \%$ & 17,791 & $13.7 \%$ \\
\hline Recursos naturales y minería & 828 & $1.6 \%$ & 1,008 & $1.1 \%$ & 629 & $0.5 \%$ & 729 & $0.6 \%$ \\
\hline Construcción & 2,881 & $5.7 \%$ & 4,562 & $5.1 \%$ & 7,233 & $5.4 \%$ & 5,614 & $4.3 \%$ \\
\hline Manufactura & 15,542 & $30.6 \%$ & 19,426 & $21.6 \%$ & 14,279 & $10.7 \%$ & 11,644 & $8.9 \%$ \\
\hline \multicolumn{9}{|l|}{ PRODUCCIÓN DE SERVICIOS } \\
\hline Total & 31,510 & $62.1 \%$ & 64,935 & $72.2 \%$ & 111,490 & $83.4 \%$ & 112,275 & $86.2 \%$ \\
\hline Comercio, transporte y servicios & 10,612 & $20.9 \%$ & 18,303 & $20.4 \%$ & 25,833 & $19.3 \%$ & 24,736 & $19.0 \%$ \\
\hline Servicios de información & 1,735 & $3.4 \%$ & 2,375 & $2.6 \%$ & 3,142 & $2.4 \%$ & 2,723 & $2.1 \%$ \\
\hline Actividades financieras & 2,212 & $4.4 \%$ & 4,843 & $5.4 \%$ & 8,227 & $6.2 \%$ & 7,597 & $5.8 \%$ \\
\hline Servicios profesionales y de negocios & 3,320 & $6.5 \%$ & 7,312 & $8.1 \%$ & 16,935 & $12.7 \%$ & 16,697 & $12.8 \%$ \\
\hline Educación y salud & 2,491 & $4.9 \%$ & 6,767 & $7.5 \%$ & 17,344 & $13.0 \%$ & 19,560 & $15.0 \%$ \\
\hline Esparcimiento y alojamiento & 3,140 & $6.2 \%$ & 6,631 & $7.4 \%$ & 12,748 & $9.5 \%$ & 13,112 & $10.1 \%$ \\
\hline Otros servicios & 978 & $1.9 \%$ & 2,637 & $2.9 \%$ & 5,467 & $4.1 \%$ & 5,353 & $4.1 \%$ \\
\hline Gobierno & 7,021 & $13.8 \%$ & 16,068 & $17.9 \%$ & 21,795 & $16.3 \%$ & 22,471 & $17.3 \%$ \\
\hline
\end{tabular}

Fuente: Elaborada por la autora con datos de Employment and Earnings, enero de 2006 y enero de 2011.

El empleo en la manufactura, aunque disminuía en términos relativos, creció numéricamente hasta 1979 alcanzando 19.4 millones. Desde entonces se han perdido más de 7.7 millones de empleos en este sector, que ha pasado de ocupar al 30.6\% de la PEA en 1955 al $21.6 \%$ en 1979 y solamente el $8.9 \%$ en 2010. En cambio, el empleo en la construcción incrementó en términos absolutos de 2.8 a 7.7 millones entre 1955 y 2006 y, con algunas fluctuaciones cíclicas, se había mantenido más o menos estable como porcentaje de la PEA (alrededor del 5\%); sin embargo, a raíz la crisis, que afectó de manera muy particular la actividad inmobiliaria, se perdieron casi 2.1 millones de empleos en la construcción entre 2006 y 2010 , y actualmente esta actividad absorbe solamente el $4.3 \%$ de la PEA.

Cabe señalar que la manufactura aportaba el $22.5 \%$ del PIB estadounidense en 1980, cuando ocupaba al 20.1\% de la PEA no agrícola, y aportaba el 11.9\% en 2009, ocupando al 9.1\% de ésta (Us Census Bureau, 1983 y 2011). Después de un ligero repunte entre 1993 y 1998, la pérdida de empleos manufactureros fue particularmente aguda a partir de 2000.

Según Wial y Friedhoff (2006), más de un tercio de los puestos perdidos en el primer lustro del siglo XXI estaban ubicados en los 7 estados 
de la región de los Grandes Lagos en el noreste de Estados Unidos. La mayoría de los afectados son hombres sin educación superior que difícilmente encontrarán otro empleo que les ofrezca el mismo nivel de ingresos y beneficios, $y$, por lo tanto, muchos de ellos han optado por abandonar la PEA. De hecho, el nivel de participación masculina en la PEA, en Estados Unidos, ha disminuido lenta pero sistemáticamente a lo largo de la segunda mitad del siglo xx y principios del siglo xxI, bajando del $86.4 \%$ en 1950 a $71.2 \%$ en 2010 (USDOL, 1984 y 2011). Pero parece que la proporción de hombres de entre 30 y 55 años - considerada la mejor etapa de la vida laboral- que han dejado de buscar empleo en los últimos años es mucho más alta que en décadas anteriores. Actualmente, el porcentaje de los que están fuera de la PEA en este rango de edad es alrededor del $13 \%$, en comparación con el $5 \%$ a finales de los años sesenta (Uchitelle y Leonhardt, 2006).

En cambio, la participación femenina en la PEA ha incrementado notablemente a lo largo de la posguerra al pasar de una tasa de $33.9 \%$ en 1950 a $58.6 \%$ en 2010 ; de tal manera que las mujeres constituyen ahora el $47.2 \%$ de la PEA. El crecimiento más acelerado se dio en las décadas de los setenta y los ochenta. En un primer momento, tal vez como búsqueda de una realización personal, catalizada por el movimiento feminista, y después como respuesta a la creciente inseguridad e inestabilidad en los ingresos familiares provocada por la reestructuración industrial y las políticas económicas neoliberales. La creciente participación femenina está relacionada también con los cambios en la estructura ocupacional, mediante la disminución de empleos industriales donde predominaba la fuerza de trabajo masculino y el incremento de empleos en algunos servicios profesionales y otros servicios y ventas donde predomina la mano de obra femenina.

La creciente participación de mujeres en la PEA y en la fuerza de trabajo ocupada es uno de los factores que contribuyó a la tendencia descendente del salario promedio en Estados Unidos a partir de 1973, que hasta la fecha no recupera el nivel de entonces en términos reales. Desafortunadamente, aun cuando tengan niveles iguales de escolaridad, las mujeres reciben salarios bastante inferiores a los de los hombres. Por otra parte, la polarización ocupacional y salarial es una de las 
características más destacadas del mercado laboral de las últimas décadas. Crecen mucho los empleos bien pagados que exigen altos niveles de escolaridad y proliferan cada vez más los empleos mal pagados que no requieren estudios universitarios. Los puestos medianamente bien pagados que no exigen estudios universitarios, como los de las industrias manufactureras más consolidadas, tienden a desaparecer.

Otro factor asociado con la disminución del salario promedio es la creciente incorporación de inmigrantes a la PEA. Andrew Sum y sus coautores afirman que, a finales del siglo $\mathrm{xx}$, el peso de los nuevos inmigrantes en el crecimiento de la fuerza laboral estadounidense fue la más alta que se había observado en los 60 años anteriores, que es desde cuando se tienen datos al respecto (Sum et al., 2002). Señalan que los 8 millones de inmigrantes nuevos ${ }^{1}$ que se incorporaron a la PEA entre 1990 y 2001, son los responsables del 50\% de su crecimiento durante este lapso. De hecho, el ritmo de crecimiento de la PEA estadounidense ha sido menguante después de la década los setenta, cuando se incrementó $29.2 \%$ debido al ingreso de las personas nacidas en la posguerra y la creciente participación de las mujeres. Durante los noventa, la PEA creció solamente $11.5 \%$, y sin los nuevos inmigrantes el crecimiento registrado hubiera sido nada más del $5 \%$. Sum y sus colaboradores aseguran también que no haber contado con los nuevos inmigrantes como parte de la PEA hubiese restringido tanto el crecimiento del empleo como el crecimiento económico en general.

Es probable que las tendencias observadas en las últimas décadas, de diversificación en la composición de la PEA y creciente polarización salarial, continúen en los próximos años. Además, la transición de más largo aliento hacia una mayor producción de servicios, en vez de bienes, continuará. El Departamento del Trabajo de Estados Unidos (USDOL por sus siglas en inglés) estima que, aproximadamente, 14.6 de los 15.3 millones de empleos que serán creados entre 2008 y 2018 pertenecerán al ámbito de provisión de servicios (USDOL, 2009b). El envejecimiento de la población incrementará considerablemente la demanda para servicios relacionados con la salud y la atención de las personas mayores. El número de mujeres en la PEA se incrementará un poco más que el de los

\footnotetext{
${ }^{1}$ Los inmigrantes considerados como «nuevos» en el trabajo citado son los que llegaron a partir de 1990.
} 
hombres, de manera que su participación aumentará de 46.5 a 46.9\%. Pero son los latinos o hispanos quienes exhibirán el crecimiento más rápido dentro de la fuerza laboral estadounidense; se estima que su participación numérica aumentará en 7.6 millones, o $33.1 \%$, a partir de 2008 cuando constituían el 14.3\% de la PEA, para alcanzar el 17.6\% en 2018 .

Las proyecciones del usDol sobre los empleos que crecerán más rápidamente durante los próximos años reflejan que habrá algunas perspectivas de empleo para inmigrantes latinos con bajos niveles de escolaridad, pero primordialmente en rubros con remuneraciones bajas (USDOL, 2009b). Más de la mitad (17) de los 30 empleos proyectados a mostrar las tasas más altas de crecimiento en los próximos años están relacionados con la atención a la salud o la investigación médica y otros cuantos están vinculados con la informática y las finanzas (ver tabla 2). La mayoría de estas ocupaciones requiere algunos años de educación superior, por lo menos dos, que es lo que se necesita para un associate degree.

TABLA 2

Ocupaciones con las más altas tasas de crecimiento, 2008-2018

\begin{tabular}{|c|c|c|c|c|}
\hline OCUPACIONES & $\%$ CAMBIO & NIVEL DE INGRESOS & ESCOLARIDAD O CAPACITACIÓN & \% LATINO EN 2008 \\
\hline Ingenieros biomédicos & $72.0 \%$ & Muy Alto & Bachelor's degree & n.d. \\
\hline Analistas de sistemas de redes y comunicación de datos & $53.4 \%$ & Muy Alto & Bachelor's degree & $6.4 \%$ \\
\hline Ayudantes en casa para atención a la salud & $50.0 \%$ & Muy Bajo & corto plazo, en el empleo & $13.1 \%$ \\
\hline Ayudantes de cuidados personales en el hogar & $46.0 \%$ & Muy Bajo & corto plazo, en el empleo & $17.4 \%$ \\
\hline Inspectores financieros & $41.2 \%$ & Muy Alto & Bachelor's degree & n.d. \\
\hline Científicos médicos excepto epidemiológos & $40.1 \%$ & Muy Alto & Doctoral degree & $2.7 \%$ \\
\hline Asistentes de médicos & $39.0 \%$ & Muy Alto & Master's degree & $9.3 \%$ \\
\hline Especialistas en cuidados de la piel & $37.9 \%$ & Bajo & post secundaria, técnica & n.d. \\
\hline Bioquímicos y biofísicos & $37.40 \%$ & Muy Alto & Doctoral degree & n.d. \\
\hline Entrenadores atléticos & $37.00 \%$ & Alto & Bachelor's degree & n.d. \\
\hline Ayudantes de fisioterapeutas & $36.3 \%$ & Bajo & corto plazo, en el empleo & $8.0 \%$ \\
\hline Técnicos dentales & $36.1 \%$ & Muy Alto & Associate degree & $5.3 \%$ \\
\hline Técnicos verterinarios & $35.8 \%$ & Bajo & Associate degree & n.d. \\
\hline Ayudantes de dentista & $35.8 \%$ & Bajo & mediano plazo, en el empleo & $17.3 \%$ \\
\hline Ingenieros en computación, software $y$ aplicaciones & $34.0 \%$ & Muy Alto & Bachelor's degree & $3.7 \%$ \\
\hline Ayudantes médicos & $34.0 \%$ & Bajo & mediano plazo, en el empleo & $15.3 \%$ \\
\hline Asistentes de fisioterapeutas & $33.3 \%$ & Alto & Associate degree & $8.0 \%$ \\
\hline Veterinarios & $33.00 \%$ & Muy Alto & Primer grado profesional & $4.1 \%$ \\
\hline \multirow{2}{*}{$\begin{array}{l}\text { Profesores de superación personal } \\
\text { inspectores excepto agricuitura, construccion, sauua y } \\
\text { transporte }\end{array}$} & $32.0 \%$ & Alto & Experiencia laboral relacionada & n.d. \\
\hline & $31.00 \%$ & Alto & largo plazo en el empleo & $6.4 \%$ \\
\hline Ayudantes de terapia ocupacional & $31.0 \%$ & Bajo & corto plazo, en el empleo & n.d. \\
\hline Ingenieros ambientales & $31.0 \%$ & Muy Alto & Bachelor's degree & n.d. \\
\hline Técnicos en farmacéutica & $30.60 \%$ & Bajo & mediano plazo, en el empleo & n.d. \\
\hline Ingenieros en computación, software $y$ aplicaciones & $30.4 \%$ & Muy Alto & Bachelor's degree & $3.7 \%$ \\
\hline Investigadores para encuestas & $30.4 \%$ & Alto & Bachelor's degree & n.d. \\
\hline Fisioterapeutas & $30.3 \%$ & Muy Alto & Master's degree & $3.5 \%$ \\
\hline Asesores financieros personales & $30.1 \%$ & Muy Alto & Bachelor's degree & $6.1 \%$ \\
\hline Técnicos en ingeniería ambiental & $30.1 \%$ & Alto & Associate degree & n.d. \\
\hline Asistentes de terapia ocupacional & $29.8 \%$ & Alto & Associate degree & n.d. \\
\hline Entrenadores de fitness e instructores de aerobics & $29.4 \%$ & Bajo & post secundaria, técnica & n.d. \\
\hline
\end{tabular}

Fuente: Elaborada por la autora con datos de U.S. Department of Labor, BLS, Employment Projections 2008-2018 y Employment and Earnings, enero de 2009. 
Para la mayoría de migrantes mexicanos, la posibilidad de acceder a estos empleos es escasa. Actualmente, la concentración de trabajadores latinos es significativa - es decir, casi igual o mayor que su participación en la población ocupada en 2008 , que fue de $14.3 \%$ - en solamente 4 de estas ocupaciones (ayudantes en casa para atención a la salud, ayudantes para cuidados personales en el hogar, ayudantes de dentista y ayudantes médicos), las cuales no requieren estudios superiores y las remuneraciones son bajas o muy bajas.

En cambio, las ocupaciones que tienen las proyecciones más altas de crecimiento numérico incluyen una gama más amplia de servicios personales, servicios en general y algunos oficios, que requieren solamente capacitación en el puesto y donde las remuneraciones son predominantemente bajas o muy bajas (ver tabla 3). La mitad de estos empleos tiene actualmente una concentración significativa (cercana a, o mayor de 14.3\%) de trabajadores latinos. Dado el cambiante perfil demográfico de la población estadounidense - bajas tasas de natalidad y altas tasas de envejecimiento- - y el relativamente bajo costo de la mano de obra de migrantes mexicanos y otros latinos con poca escolaridad, es probable que Estados Unidos ofrecerá un número creciente de empleos para ellos en años venideros, sobre todo una vez que la recuperación económica incipiente de finales de 2010 logre tener un impacto sobre el empleo. Sin embargo, para tener una idea más clara de lo que implica la proliferación de este tipo de empleos de bajos salarios que parecen no tener fin -muchos de los cuales han sido etiquetados como «nichos del mercado laboral para inmigrantes»-, es necesario analizar con más detalle los patrones de inserción de los migrantes latinos en el mercado laboral estadounidense, un mercado que en las últimas décadas se ha tornado cada vez más segmentado y estratificado. 
TABLA 3

Mediana del ingreso de los hombres que trabajan tiempo completo

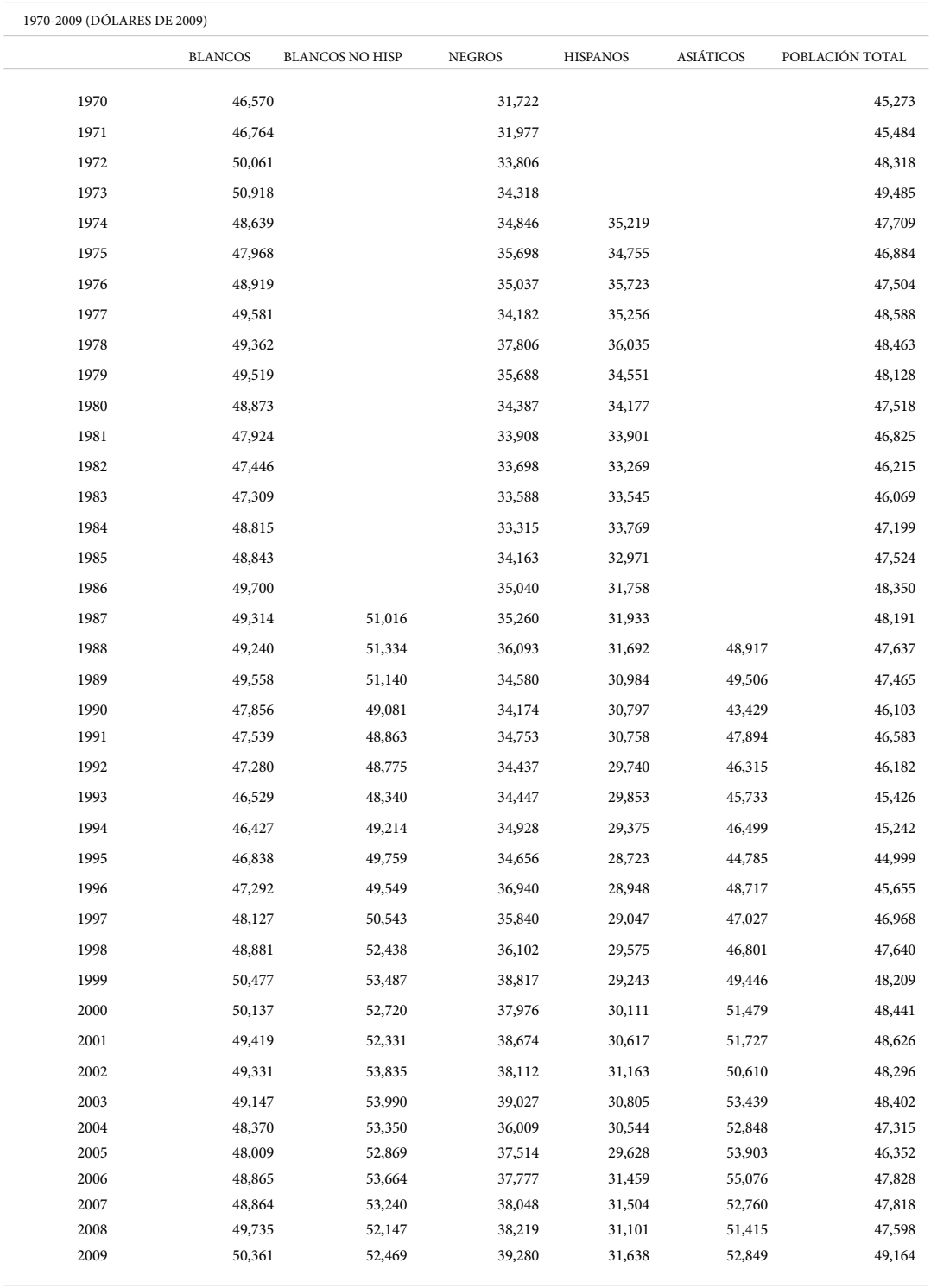

Fuente: March Current Population Survey, Historical Income Tables-Persons, http://www.census. gov/hhes/www/income/data/historical/people/index.html (Consultada el 15 febrero de 2011). 


\section{MEXICANOS Y OTROS LATINOS EN EL MERCADO LABORAL DE ESTADOS UNIDOS}

Puesto que la motivación principal para migrar a Estados Unidos es conseguir un empleo que paga en dólares, no es sorprendente que los mexicanos sean el grupo con la tasa de participación más alta en la PEA en dicho país, que fue de $67.7 \%$ en 2010 (USDOL, 2011). Como se puede ver en la gráfica 1, la tasa de $82.2 \%$ para los hombres mexicanos es bastante mayor que la de cualquier otro grupo. Aunque la tasa para las mujeres, de $54.1 \%$, es un poco más baja que la de algunos otros grupos, resulta mucho más alta que la tasa de participación en la PEA para las mujeres en México, que es alrededor del $38 \%$.

Cabe señalar que, durante las últimas tres décadas o más, las tasas de desempleo para los latinos de origen mexicano y los latinos en general han sido mayores que las de los blancos ${ }^{2}$ y menores que las de los negros. Las tasas de desempleo reflejan los altibajos de la actividad económica general, subiendo y bajando en contraposición a esta.

GRÁFICA 1

Tasas de participación en la PEA 2010

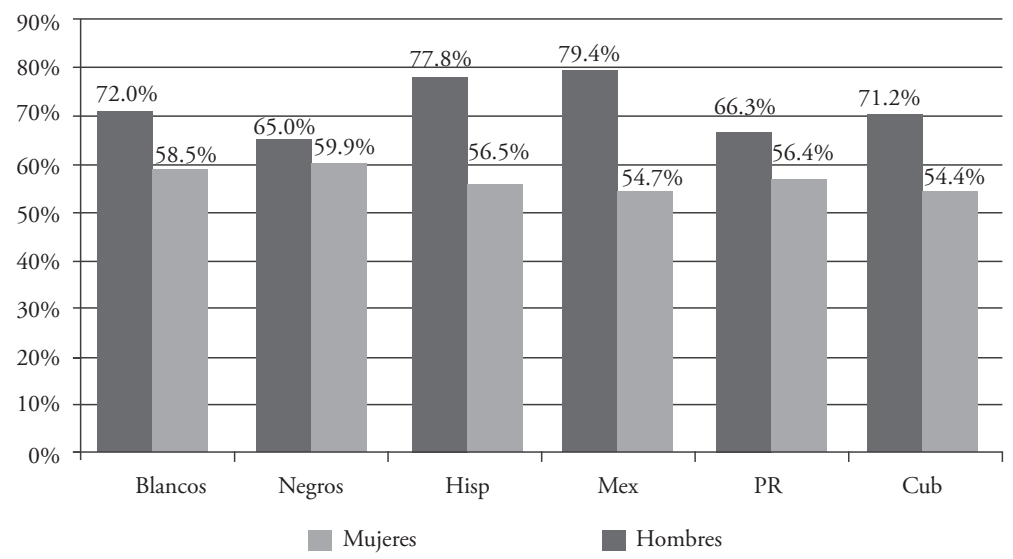

Fuente: Elaborada por la autora con datos de Employment and Earnings 2011.

\footnotetext{
${ }^{2}$ La tasa de desempleo de los blancos no hispanos es más baja que la cifra aquí indicada, que incluye también a gran número de hispanos dentro del conjunto de blancos.
} 
En general, la tasa de desempleo de los mexicanos es más baja que la de los puertorriqueños y más alta que la de los cubanos. Los cubanos suelen tener tasas de desempleo ligeramente inferiores a las de la población blanca total (que como se ha señalado incluye a la mayoría de los hispanos). Lo que llama la atención en 2010 (ver gráfica 2) es el alto desempleo entre los hombres cubanos, que inclusive supera la tasa de los varones mexicanos, y las tasas extremadamente altas para hombres negros y puertorriqueños.

GRÁFICA 2

Tasas de desempleo 2010

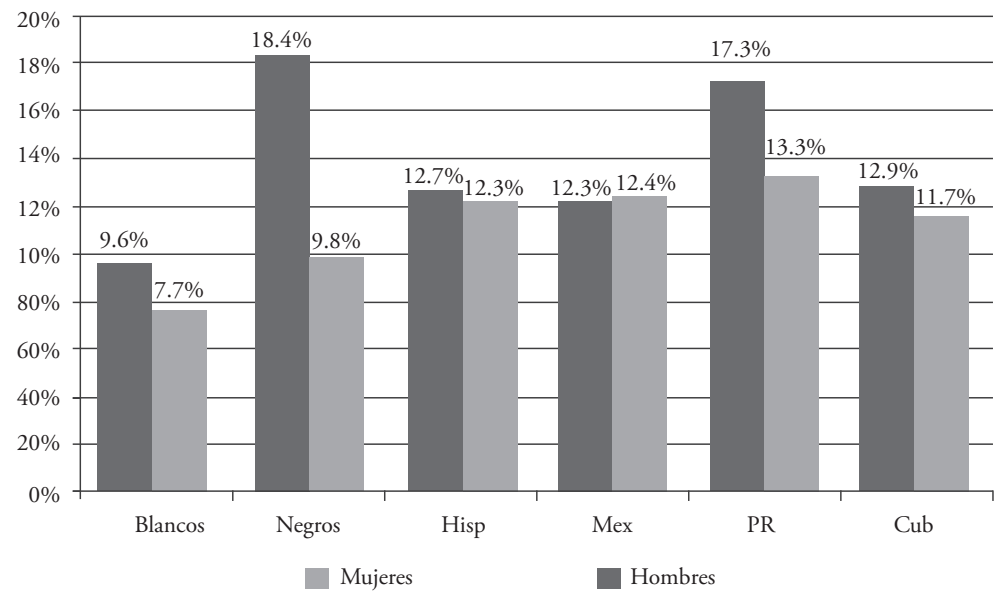

Fuente: Elaborada por la autora con datos de Employment and Earnings 2011.

Por otra parte, no es extraño que inmigrantes recientes ocupen los puestos de trabajo menos deseables con los salarios más bajos de Estados Unidos, que, sin embargo, representan mucho más de lo que podrían ganar en sus países de origen. Tal ha sido la experiencia de la gran mayoría de los migrantes mexicanos, dados sus bajos niveles de escolaridad y sus pocos conocimientos del inglés. Estas desventajas en términos de escolaridad (que veremos más adelante) persisten entre las segundas y terceras generaciones y repercuten en las oportunidades laborales de muchos latinos de origen mexicano que hayan nacido en aquel país.

Datos del Mexican Migration Project (MMP) reflejan cómo evolucionaron las oportunidades de empleo para migrantes mexicanos en Estados Unidos durante las últimas décadas del siglo $\mathrm{xx}$ (Durand $\mathrm{y}$ 
Massey, 1982-2011). ${ }^{3}$ El cambio más notable fue que la agricultura dejó de ser la actividad principal entre los trabajadores migrantes. Entre los entrevistados que hicieron su último viaje antes de 1981, el $53.2 \%$ encontró empleo en actividades agrícolas en Estados Unidos. Pero solamente el $24.4 \%$ de los que migraron después de 1991 tuvo este tipo de empleos. Este cambio refleja, en primer lugar, una transformación de la estructura ocupacional estadounidense y también el hecho de que un número creciente de migrantes mexicanos provienen de entornos urbanos. En épocas recientes, más y más migrantes han encontrado empleo en la manufactura ligera, las industrias agroalimentarias y los servicios. El porcentaje de los entrevistados por el MMP que trabajaban en la manufactura ligera y la construcción aumentó de $19.4 \%$, para el periodo anterior a 1981, a $25 \%$, a partir de 1990 . Al mismo tiempo, el empleo en el sector de servicios creció de 9.9 a 17.8\%; el empleo en ventas y puestos técnicos o administrativos aumentó de 2.5 a 5.3\%; y el porcentaje de obreros calificados creció de 9.0 a $18.1 \%$.

Datos del USDOL indican que actualmente la PEA de origen mexicano, es decir, migrantes y sus descendientes nacidos en Estados Unidos, se distribuye con cierta uniformidad entre 3 de las 5 principales categorías ocupacionales: $19 \%$ en recursos naturales, construcción y mantenimiento; $18.2 \%$ en producción, transporte y movimiento de materiales; y $19.9 \%$ en ventas y ocupaciones de oficinistas (USDOL, 2011). Su participación en el rubro de gerencia, profesionistas y ocupaciones relacionadas (16.1\%) es más baja que la de cualquier otro grupo étnico o racial. Al mismo tiempo, su participación como trabajadores en el sector de servicios es el más alto, superando ligeramente a la de los afroamericanos y también la de los puertorriqueños y cubanos. Solamente el 3.0\% de los trabajadores mexicanos se emplean en actividades de agricultura, pesca y silvicultura -que a partir de 2004 ya no aparece como categoría independiente-, un porcentaje mucho más alto que el de cualquier otro grupo (ver tabla 4 ).

\footnotetext{
${ }^{3}$ La autora agradece a Marcela Osnaya su apoyo para procesar los datos del Mexican Migration Project MMP93. Para poder observar cambios en los patrones ocupacionales, establecimos tres grupos: 1) los que tuvieron su última estancia en EU antes de 1981;2) los que estuvieron por última vez entre 1981 y 1990; y 3) los que han estado en EU después de 1990.
} 
TABLA 4

Distribución ocupacional por grupos de población, 2010

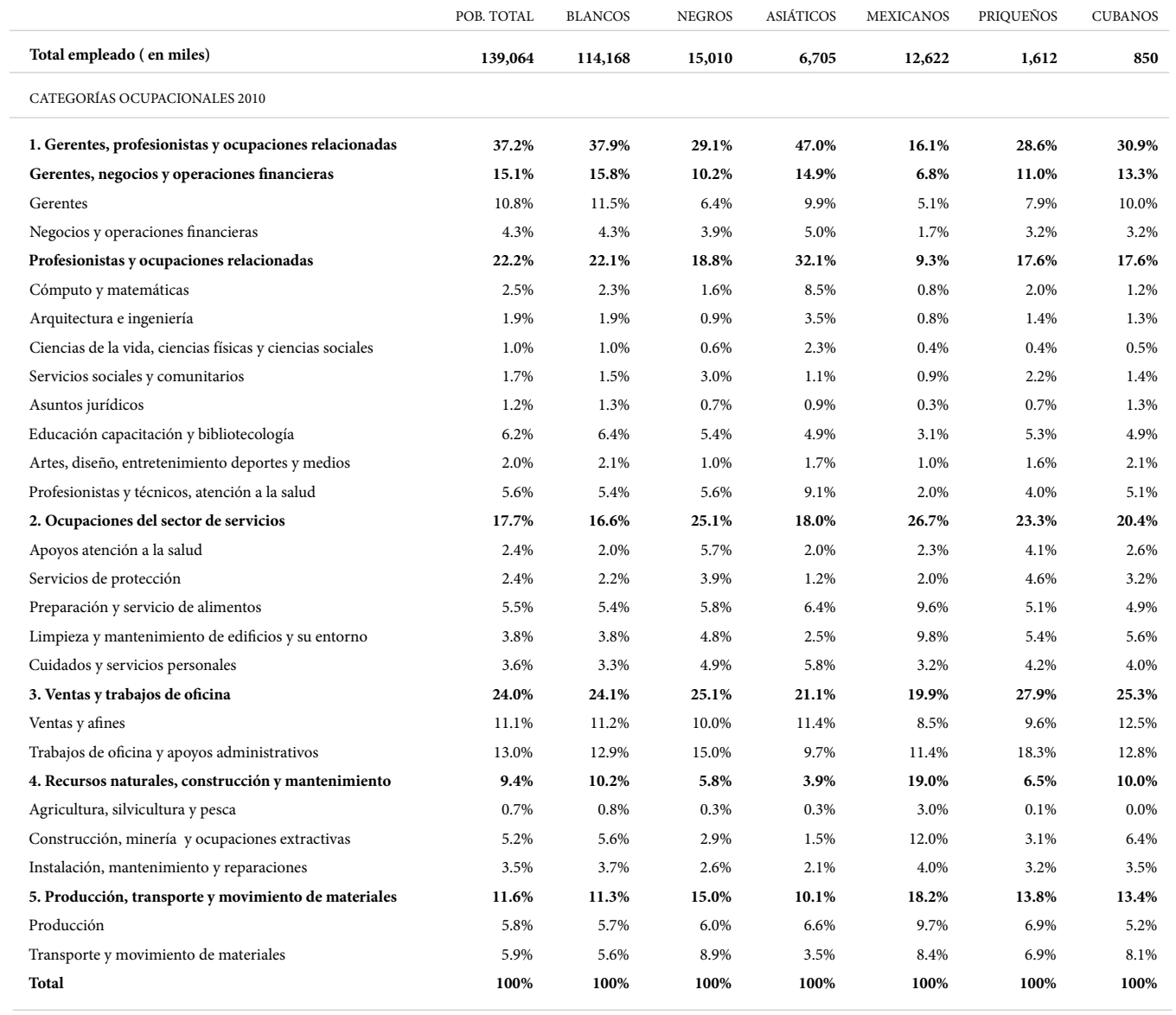

Fuente: Elaborada por la autora con datos de Employment and Earnings. 
El 9.3\% de los mexicanos se ocupa en puestos de profesionista y ocupaciones afines. Un número similar (9.7\%) trabaja en la manufactura, y $12 \%$ está empleado en trabajos de construcción y extracción; en estos dos rubros hay algunos puestos bien remunerados para trabajadores altamente calificados y con mucha experiencia, pero la mayoría son puestos de bajos salarios y baja calificación. El 11.4\% tiene puestos de oficinistas y apoyo administrativo. En esta categoría hay muchos rubros donde predominan las mujeres y los salarios tienden a ser bajos. Lo mismo sucede en el área de ventas, que absorbe al 8.5\% de los trabajadores mexicanos. El 9.6 y 9.8\%, respectivamente, trabaja preparando y sirviendo alimentos o limpiando y manteniendo edificios y jardines, ocupaciones con salarios muy bajos.

Dentro de cada una de las categorías más generales, los mexicanos y otros latinos suelen encontrarse concentrados en unos cuantos rubros: ciertas ramas específicas de la manufactura ligera, más que de la pesada; servicios de limpieza y mantenimiento de edificios y jardines; preparación y servicio de alimentos; cajeros en tiendas de autoservicio y ventas de menudeo; trabajos especializados de albañilería, por mencionar algunos. Los datos por industria (ver tabla 5) revelan que algunos sectores dependen cada vez más de la mano de obra latina (USDOL, 1991, 2008 y 2011).

Entre 1990 y 2010 , el porcentaje de trabajadores latinos en la PEA se incrementó de 7.5 a 14.3\%, a la vez que en actividades de apoyo para la agricultura y silvicultura creció de 15.4 a $35.5 \%$. En servicios de diseño y mantenimiento de jardines creció de 25.2 a $41.5 \%$; en la confección aumentó de 22.6 a 34.8\%; en el rubro de servicios para edificios y viviendas se incrementó de 18 a 35.6\%; en servicios de lavandería y tintorería creció de 14.6 a $28.5 \%$; y en el servicio doméstico privado pasó de 17.6 a $39.5 \%$. En la rama general de alimentos procesados el crecimiento fue de 14.1 a $27.6 \%$ y resultó aún más pronunciado en algunos subsectores como matanza y procesamiento de animales (de 17.0 al $38.1 \%$ ) y la panadería industrial, no de menudeo (de 13.0 a 31.8\%). Todo eso sucedió después de haber alcanzado porcentajes aún mayores en algunas de estas industrias en 2007. 
TABLA 5

Industrias con alta concentración de trabajadores latinos

\begin{tabular}{|c|c|c|c|c|c|c|c|}
\hline \multicolumn{8}{|l|}{ ORDENADAS POR \% LATINO EN 2007} \\
\hline & $\begin{array}{l}\text { \% LATINO } \\
1990\end{array}$ & $\begin{array}{l}\text { \% LATINO } \\
2007\end{array}$ & $\begin{array}{l}\text { \% AUMENTO } \\
1990-2007\end{array}$ & \# LATINO 2007 & $\begin{array}{l}\text { \% LATINO } \\
2009\end{array}$ & $\begin{array}{l}\text { \% LATINO } \\
2010\end{array}$ & \# LATINO 2010 \\
\hline Total empleado, 16 años y más & $7.5 \%$ & $14.0 \%$ & $86.7 \%$ & $20,446,580$ & $14.0 \%$ & $14.3 \%$ & $19,886,152$ \\
\hline \multicolumn{8}{|l|}{ INDUSTRIA } \\
\hline Servicios de diseño y mantenimiento de jardines & $25.2 \%$ & $43.7 \%$ & $73.4 \%$ & 560,234 & $38.9 \%$ & $41.5 \%$ & 489,700 \\
\hline Confección de ropa & $22.6 \%$ & $39.6 \%$ & $75.2 \%$ & 134,244 & $37.3 \%$ & $34.8 \%$ & 79,692 \\
\hline Actividades de apoyo, agricultura y silvicultura & $15.4 \%$ & $37.1 \%$ & $140.9 \%$ & 52,311 & $29.7 \%$ & $35.5 \%$ & 68,515 \\
\hline Matanza y procesamiento de animales & $17.0 \%$ & $35.2 \%$ & $107.1 \%$ & 167,904 & $39.6 \%$ & $38.1 \%$ & 179,832 \\
\hline Servicio doméstico privado & $17.6 \%$ & $34.8 \%$ & $97.7 \%$ & 282,924 & $37.8 \%$ & $39.5 \%$ & 263,465 \\
\hline Servicios para edificios $y$ viviendas & $18.0 \%$ & $33.1 \%$ & $83.9 \%$ & 438,575 & $33.9 \%$ & $35.6 \%$ & 492,348 \\
\hline Panaderías, no de menudeo & $13.0 \%$ & $31.7 \%$ & $143.8 \%$ & 69,423 & $32.8 \%$ & $31.8 \%$ & 66,144 \\
\hline Lavanderías y tintorerías & $14.6 \%$ & $31.2 \%$ & $113.7 \%$ & 115,128 & $27.7 \%$ & $28.5 \%$ & 98,895 \\
\hline Fábricas de alfombras y tapetes & $10.1 \%$ & $29.4 \%$ & $191.1 \%$ & 18,522 & $19.2 \% *$ & $49.0 \%$ & 28,910 \\
\hline Producción agrícola & $19.5 \%$ & $28.8 \%$ & $47.7 \%$ & 258,048 & $28.5 \%$ & $30.6 \%$ & 301,104 \\
\hline Lavado de automóviles & $22.5 \%$ & $27.8 \%$ & $23.6 \%$ & 42,812 & $36.6 \%$ & $34.8 \%$ & 55,332 \\
\hline Conservas de frutas y vegetales (specialty foods) & $21.0 \%$ & $27.6 \%$ & $31.4 \%$ & 45,816 & $28.4 \%$ & $30.0 \%$ & 49,800 \\
\hline Productos textiles y de piel & $20.2 \%$ & $27.0 \%$ & $33.7 \%$ & 217,350 & $28.2 \%$ & $29.0 \%$ & 171,680 \\
\hline Alimentos procesados & $14.1 \%$ & $26.7 \%$ & $89.4 \%$ & 427,200 & $28.8 \%$ & $27.6 \%$ & 463,404 \\
\hline Construcción & $8.5 \%$ & $25.3 \%$ & $197.6 \%$ & $2,999,568$ & $23.5 \%$ & $24.4 \%$ & $2,214,788$ \\
\hline Alojamiento para viajeros & $15.2 \%$ & $24.8 \%$ & $63.2 \%$ & 340,752 & $22.5 \%$ & $24.4 \%$ & 319,396 \\
\hline
\end{tabular}

* Dato de 2008; no hay dato disponible para 2009 porque el número total de empleados es menor de 50,000 .

Fuente: Employment and Earnings, enero de 1991, 2008, 2010 y 2011.

Pero los incrementos más espectaculares se dieron en la construcción y en la fabricación de alfombras. El porcentaje en la construcción creció de $8.5 \%$ en 1990 a $25.3 \%$ en 2007 con casi 3 millones de trabajadores latinos. Dado el fuerte impacto de la crisis en este rubro, para 2010, el número de latinos había bajado a 2.1 millones, que son el $24.4 \%$ del total ahora empleado en dicha industria. En la producción de alfombras y tapetes, la participación de la mano de obra latina incrementó de $10.1 \%$ en 1990 a $29.4 \%$ en 2007; sufrió una caída muy fuerte, al $19.2 \%$ en 2008 y después un incremento espectacular para alcanzar el $49 \%$ del total ocupado en 2010. De todas formas, el número de personas ocupadas en esta industria es muy pequeña, solamente un 59 mil en 2010. Dalton, Georgia, etiquetada como la ciudad de las alfombras, es el centro más importante para esa industria en Estados Unidos y ahora los latinos constituyen más de un tercio de la población local.

La concentración ocupacional e industrial de los latinos se entrelaza con la concentración geográfica, muy pronunciada entre ellos. Al iniciar 
el siglo Xxi, el $75 \%$ de la población latina estaba ubicada en solamente 7 estados; sin embargo, un grupo de estados del sureste - cuya población latina es todavía bastante pequeña - registró tasas de crecimiento espectaculares - de más de 200 a casi $400 \%$ entre 1990 y 2000 - en el número de latinos que residen allí, precisamente por las oportunidades de empleo que existen para ellos. Constantemente mexicanos y otros latinos son reclutados para llenar puestos que los residentes locales desdeñan en las empacadoras de carne, procesadoras de pollos o fábricas de alfombras. Para consolidar un nicho de mercado de este tipo, parece que solamente se necesita una afluencia de inmigrantes latinos y trabajos que casi nadie más quiere desempeñar o salarios que otros no aceptarían.

Este hecho es también muy claro en el caso de los trabajos agrícolas en estados como California, Texas y Oregon. La demanda de mano de obra para desempeñar estos trabajos no deseables y con remuneraciones bajas creció marcadamente a finales del siglo $\mathrm{xx}$, al mismo tiempo que llegaron las nuevas oleadas de inmigrantes provenientes de México y otros países latinoamericanos, más que dispuestos a realizarlos.

Desafortunadamente, las estadísticas más desagregadas en términos ocupacionales solamente registran el porcentaje de «hispanos o latinos» con respecto al total de personas empleadas en cada categoría; no distinguen entre los diferentes grupos que componen la población hispana, de tal manera que no podemos analizar aquí los diferentes perfiles ocupacionales con mayor detalle. Lo que se puede observar a partir de la información disponible es el porcentaje y, por ende, el número de trabajadores hispanos en cada rubro de la lista de categorías ocupacionales detalladas que publica el USDOL (2010). Al respecto, cabe señalar que dada la preponderancia de los mexicanos en el conjunto - cerca de dos tercios de los trabajadores hispanos son de origen mexicano- y el hecho de que las divergencias de los otros dos grupos principales, cubanos y puertorriqueños, son a menudo contrapuestos entre sí, los datos para el total de la población hispana nos pueden dar una buena aproximación de la inserción laboral de los mexicanos.

En la tabla 6 se pueden observar las ocupaciones en las que se emplean los números más altos de trabajadores latinos (más de 170 mil) a 
nivel nacional. La mayoría son empleos poco calificados y de salarios bajos que no exigen estudios superiores. Solamente 5 de las ocupaciones en la lista tienen un salario promedio cercano a, o por encima de, la mediana general, y las dos que están bien remuneradas (por encima de la mediana general) tienen una participación baja de trabajadores latinos - menor al 14.3\%, que es su proporción dentro de la PEA totalen términos relativos, es decir, como porcentaje del total de personas empleadas en dicha categoría. Todas las que tienen una concentración más alta del 14.3\% de latinos registraron medianas salariales inferiores a la mediana general de 739 dólares por semana en 2009.

TABLA 6

Ocupaciones con el mayor número de trabajadores latinos, 2009

\begin{tabular}{|c|c|c|c|}
\hline OCPACIONES 2009 & \# LATINO 2009 & \% LATINO 2009 & $\begin{array}{l}\text { MEDIANA INGRESO } \\
\text { SEMANAL (DÓLARES } \\
\text { CORRIENTES) }\end{array}$ \\
\hline Total, 16 años y más & $19,582,780$ & $14.0 \%$ & $\$ 739$ \\
\hline Cocineros & 643,284 & 32.1 & $\$ 389$ \\
\hline Intendentes y limpiadores de edificios & 631,806 & 29.4 & $\$ 473$ \\
\hline Obreros construcción & 630,734 & 44.2 & $\$ 596$ \\
\hline Sirvientes y servicios de limpieza & 595,476 & 41.7 & $\$ 377$ \\
\hline Choferes/repartidores y choferes camiones de carga & 589,237 & 18.7 & $\$ 685$ \\
\hline Cajeros & 525,632 & 17.2 & $\$ 378$ \\
\hline Mantenimiento de jardines y entornos exteriores & 466,488 & 39.6 & $\$ 437$ \\
\hline Ventas menudeo & 445,480 & 14 & $\$ 523$ \\
\hline $\begin{array}{l}\text { Obreros, carga, inventarios y movimiento de } \\
\text { materiales (manual) }\end{array}$ & 373,833 & 21.9 & $\$ 502$ \\
\hline Supervisores/gerentes de vendedores al menudeo & 341,033 & 10.3 & $\$ 688$ \\
\hline Trabajos agrícolas diversos & 311,217 & 45.7 & $\$ 396$ \\
\hline Carpinteros & 305,888 & 24.2 & $\$ 662$ \\
\hline Meseros/meseras & 300,750 & 15 & $\$ 383$ \\
\hline Secretarias y ayudantes administrativos & 292,192 & 9.2 & $\$ 620$ \\
\hline Representantes servicios al cliente & 290,472 & 15.6 & $\$ 592$ \\
\hline Ayudantes de enfermeras, psiquiátricos y en el hogar & 248,248 & 12.4 & $\$ 438$ \\
\hline Almacenistas & 244,475 & 17.5 & $\$ 483$ \\
\hline Otros gerentes diversos & 240,426 & 7.4 & $\$ 996$ \\
\hline Trabajadores, cuidado de niños & 237,728 & 18.4 & $\$ 367$ \\
\hline Pintores construcción y mantenimiento & 208,278 & 39.9 & $\$ 529$ \\
\hline Profesores de primaria, secundaria y preparatoria & 203,202 & 7.1 & $\$ 918$ \\
\hline Trabajadores de la producción, no especificados & 192,873 & 23.9 & $\$ 591$ \\
\hline Recepcionistas e informes & 192,827 & 15.1 & $\$ 517$ \\
\hline Ayudantes para cuidados personales en el hogar & 175,940 & 19 & $\$ 409$ \\
\hline $\begin{array}{l}\text { Supervisores de trabajadores de oficina y apoyos } \\
\text { administrativos }\end{array}$ & 174,624 & 10.7 & $\$ 740$ \\
\hline Preparación de alimentos & 170,544 & 22.8 & $\$ 376$ \\
\hline
\end{tabular}

Fuente: Elaborada por la autora con datos de Employment and Earnings, enero de 2010. 
La tabla 7 muestra las ocupaciones con la concentración más alta de trabajadores latinos, como porcentaje del total de empleados en el rubro; incluye todas las ocupaciones en las que la concentración de latinos es mayor a $29 \%$, que es dos veces más que su participación en el empleo total, que fue de $14.3 \%$ en 2009. Esta lista solamente coincide de forma parcial con la anterior, puesto que muchas ocupaciones con un alto porcentaje de latinos emplean menos personas, en términos absolutos, que las de aquella lista. En todas estas ocupaciones con una alta concentración de latinos, la mediana del ingreso está por debajo de la mediana general (de 739 dólares semanales), aunque 3 de estas 27 ocupaciones sí tienen una mediana bastante cercana a la general.

TABLA 7

Ocupaciones con los porcentajes más altos de trabajadores latinos, 2009

\begin{tabular}{|c|c|c|c|}
\hline OCPACIONES 2009 & $\%$ LATINO & \# LATINO & $\begin{array}{l}\text { MEDIANA INGRESO } \\
\text { SEMANAL (DÓLARES } \\
\text { CORRIENTES) }\end{array}$ \\
\hline Total, 16 años y más & $14.0 \%$ & $19,582,780$ & $\$ 739$ \\
\hline Clasificadores productos agrícolas & 58.0 & 40,600 & $\$ 408$ \\
\hline Instaladores tabla roca y plafones & 56.6 & 96,220 & $\$ 590$ \\
\hline Trabajadores cemento, acabados, concreto y terrazzo & 51.5 & 38,110 & $\$ 631$ \\
\hline Ayudantes en la construcción & 48.6 & 31,104 & $\$ 507$ \\
\hline Instalación de techos & 47.7 & 103,032 & $\$ 600$ \\
\hline Trabajos agrícolas diversos & 45.7 & 311,217 & $\$ 396$ \\
\hline Operadores de máquinas de envasado y llenado & 45.1 & 106,887 & $\$ 447$ \\
\hline Instaladores de alfombras pisos y azulejos & 44.5 & 99,235 & $\$ 586$ \\
\hline Obreros de la construcción & 44.2 & 630,734 & $\$ 596$ \\
\hline Trabajadores empaque y envoltura manual & 43.7 & 165,186 & $\$ 408$ \\
\hline Sirvientes y servicios de limpieza & 41.7 & 595,476 & $\$ 377$ \\
\hline Operadores máquinas de coser & 41.7 & 82,149 & $\$ 385$ \\
\hline Pintores construcción y mantenimiento & 39.9 & 208,278 & $\$ 529$ \\
\hline Mantenimiento de jardines y entornos exteriores & 39.6 & 466,488 & $\$ 437$ \\
\hline Lavadores de platos & 39.5 & 103,885 & $\$ 334$ \\
\hline Limpiadores de vehículos y equipo & 37.1 & 117,236 & $\$ 421$ \\
\hline Trabajadores diversos en medios y comunicaciones & 37.0 & 34,780 & $\$ 680$ \\
\hline $\begin{array}{l}\text { Carniceros y procesadores de carne, pollo y pescado } \\
\text { rtancnadores, Inaustria conteccion, textules y materiales }\end{array}$ & 35.9 & 108,418 & $\$ 491$ \\
\hline relacionados & 35.7 & 19,635 & n.d. \\
\hline Panaderos & 32.7 & 63,111 & $\$ 455$ \\
\hline Preparación de alimentos por tandas & 32.5 & 28,275 & $\$ 493$ \\
\hline Cocineros & 32.1 & 643,284 & $\$ 389$ \\
\hline Albañiles especializados, tabique, block y piedra & 31.3 & 47,263 & $\$ 701$ \\
\hline Trabajadores lavanderías y tintorerías & 30.6 & 58,752 & $\$ 391$ \\
\hline Hojalatería automotríz y similares & 30.5 & 49,715 & $\$ 661$ \\
\hline Cortadores industriales & 29.9 & 24,219 & $\$ 511$ \\
\hline Intendentes y limpiadores de edificios & 29.4 & 631,806 & $\$ 473$ \\
\hline
\end{tabular}

Fuente: Elaborada por la autora con datos de Employment and Earnings, enero de 2010. 
Los trabajadores agrícolas ameritan una mención especial, puesto que, a partir de 2004 (con los datos para 2003), el rubro de «farming, fishing and forestry occupations» ha sido eliminado como categoría general para ser incorporado como subcategoría del rubro «natural resources, construcción, and maintennance occupations». Por una parte, el número de personas registradas como empleados en dicho rubro disminuyó marcadamente de 3.4 millones en 2002 a menos de 1 millón (926 mil) en 2009. Además, la ocupación de «farm workers» ya ni siquiera aparece. Aplicar el porcentaje indicado para latinos (40.7\%) al total de empleados del rubro arriba señalado da como resultado 376,882 trabajadores latinos para las diversas actividades comprendidas. Por otra parte, las cifras por industria indican que había 916 mil personas dedicas al rubro de «crop production» en 2009, y que el $28.5 \%$ de estas son hispanos, eso es unos 261,060 . Se podría tomar estas dos cifras $(261,060$ y 376,882$)$ como un rango indicativo del número de latinos, sin duda principalmente mexicanos, que se encuentran dedicados de manera más o menos permanente a las actividades agrícolas en Estados Unidos. Es muy probable que los miles de trabajadores itinerantes - dedicados a la cosecha de una gran variedad de productos agrícolas- que permanecen solamente unas cuantas semanas en el mismo lugar y la gran mayoría de los cuales seguramente son indocumentados, no están debidamente contabilizados en estas cifras.

Por otra parte, cabe señalar que casi todas las ocupaciones mencionadas en la tabla 7 , de las ocupaciones con las más altas concentraciones de trabajadores latinos, aparecen también en la lista de ocupaciones con altos porcentajes de trabajadores «no autorizadas» contempladas en el estudio de Passel (2006:12) del Pew Hispanic Center; él estima que más de la mitad (56\%) de los inmigrantes indocumentados son mexicanos y que el 80 u $85 \%$ de los mexicanos que tenían menos de 10 años en Estados Unidos, a fines de 2006, se encontraban en esta situación.

Los trabajadores indocumentados son aun más vulnerables que los demás por la irregularidad de su estatus migratorio. Pero la situación laboral de la mayoría de los latinos poco calificados es precaria, de por sí, debido a cambios recientes en las condiciones laborales generales impuestas como respuesta a la competencia y la globalización, dando paso a un mercado laboral cada vez más segmentado y estratificado. 


\section{NIVELES DE INGRESOS Y SITUACIÓN SOCIOECONÓMICA DE LOS} MIGRANTES LATINOS

Las nuevas prácticas y estrategias empresariales asociadas con la reestructuración industrial de las últimas tres décadas generaron mayor inestabilidad e inseguridad en el empleo para la mayoría de los trabajadores, minando su poder de negociación. A finales del siglo xx y principios del xxI, familias de profesionistas y de trabajadores y empleados calificados - que respondieron a las vicisitudes de los setenta y ochenta con la incorporación creciente de las mujeres a la PEA- hicieron frente a las nuevas exigencias del mercado con más horas de trabajo; por ende, se volvieron demandantes de más bienes de consumo y servicios personales proporcionados por trabajadores menos calificados cuya remuneración ha disminuido marcadamente, en términos relativos, respecto a la del resto de la población, no obstante la demanda creciente que había hasta 2007 para las labores que desempeñan.

Los nuevos «nichos de empleos para inmigrantes» —que ofrecen condiciones de trabajo y salarios inaceptables para la mayoría de los estadounidenses - crecieron a la par de la oferta aparentemente inagotable de recién llegados que reciben lo que para ellos representa, generalmente, de 10 a 15 veces, o más, de lo que podrían ganar en sus países de origen. Aun así, la mayoría se encuentra relegada a los estratos inferiores del espectro socioeconómico en Estados Unidos. «Aunque los trabajadores latinos constituyen una proporción creciente de la fuerza de trabajo de aquel país persisten entre ellos altos índices de pobreza y desempleo así como bajos ingresos» (Thomas-Breitfeld, 2003).

No deja de llamar la atención el deterioro salarial, en términos relativos, en casi todas las ocupaciones donde hay alta concentración de trabajadores latinos (ver tabla 8). El declive es particularmente notorio en el caso de ciertos oficios de la construcción (albañiles especializados en tabique, block y piedra; instaladores de tablarroca y plafones; trabajadores de cemento, acabados de concreto y terrazzo) donde, en 1990, la mediana del ingreso semanal era todavía igual o mayor que la mediana general, y para 2009 era ya bastante inferior a la mediana semanal general (USDOL, 1991 y 2010). Cabe señalar que este deterioro fue muy evidente antes de la crisis que inició a fines de 2007. Durante los últimos 20 años, en general, los trabajadores latinos han experimentado un deterioro salarial frente a otros grupos de la población estadounidense. 
TABLA 8

Comparación de medianas del ingreso para ocupaciones con alto porcentaje de latinos, 1990 y 2009

\begin{tabular}{|c|c|c|c|c|c|c|}
\hline OCUPACIONES 2009 & $\begin{array}{l}\text { MEDIANA } 1990 \\
\text { (DÓLARES } \\
\text { CORRIENTES) }\end{array}$ & $\begin{array}{c}\text { \% DE LA } \\
\text { MEDIANA } \\
\text { GENERAL } 1990\end{array}$ & $\begin{array}{l}\text { MEDIANA } 2009 \\
\text { (DÓLARES } \\
\text { CORRIENTES) }\end{array}$ & $\begin{array}{c}\text { \% DE LA } \\
\text { MEDIANA } \\
\text { GENERAL } 2009\end{array}$ & \% LATINO 2009 & \# LATINO 2009 \\
\hline Total, 16 años y más & $\$ 415$ & $100 \%$ & $\$ 739$ & $100 \%$ & $14.0 \%$ & $19,582,780$ \\
\hline Clasificadores productos agrícolas & n.d. & n.d. & $\$ 408$ & $55 \%$ & 58.0 & 40,600 \\
\hline Instaladores tabla roca y plafones & $\$ 440$ & $106 \%$ & $\$ 590$ & $80 \%$ & 56.6 & 96,220 \\
\hline Trabajadores cemento, acabados, concreto y terrazzo & $\$ 414$ & $100 \%$ & $\$ 631$ & $85 \%$ & 51.5 & 38,110 \\
\hline Ayudantes en la construcción & $\$ 272$ & $66 \%$ & $\$ 507$ & $69 \%$ & 48.6 & 31,104 \\
\hline Instalación de techos & $\$ 341$ & $82 \%$ & $\$ 600$ & $81 \%$ & 47.7 & 103,032 \\
\hline Trabajos agrícolas diversos & $\$ 250$ & $60 \%$ & $\$ 396$ & $54 \%$ & 45.7 & 311,217 \\
\hline Operadores de máquinas de envasado y llenado & $\$ 264$ & $64 \%$ & $\$ 447$ & $60 \%$ & 45.1 & 106,887 \\
\hline Instaladores de alfombras pisos y azulejos & $\$ 376$ & $91 \%$ & $\$ 586$ & $79 \%$ & 44.5 & 99,235 \\
\hline Obreros de la construcción & $\$ 347$ & $84 \%$ & $\$ 596$ & $81 \%$ & 44.2 & 630,734 \\
\hline Trabajadores empaque y envoltura manual & $\$ 258$ & $62 \%$ & $\$ 408$ & $55 \%$ & 43.7 & 165,186 \\
\hline Sirvientes y servicios de limpieza & $\$ 220$ & $53 \%$ & $\$ 377$ & $51 \%$ & 41.7 & 595,476 \\
\hline Operadores máquinas de coser & $\$ 292$ & $70 \%$ & $\$ 385$ & $52 \%$ & 41.7 & 82,149 \\
\hline Mantenimiento de jardines y entornos exteriores & $\$ 267$ & $67 \%$ & $\$ 437$ & $59 \%$ & 39.6 & 466,488 \\
\hline Lavadores de platos & n.d. & n.d. & $\$ 334$ & $45 \%$ & 39.5 & 103,885 \\
\hline Limpiadores de vehículos y equipo & $\$ 249$ & $60 \%$ & $\$ 421$ & $57 \%$ & 37.1 & 117,236 \\
\hline Trabajadores diversos en medios y comunicaciones & n.d. & & $\$ 680$ & $92 \%$ & 37.0 & 34,780 \\
\hline Carniceros y procesadores de carne, pollo y pescado & $\$ 314$ & $76 \%$ & $\$ 491$ & $66 \%$ & 35.9 & 108,418 \\
\hline Planchadores, industria confección, textiles y materiales relacionados & $\$ 222$ & $53 \%$ & n.d. & n.d. & 35.7 & 19,635 \\
\hline Panaderos & $\$ 304$ & $73 \%$ & $\$ 455$ & $62 \%$ & 32.7 & 63,111 \\
\hline Preparación de alimentos por tandas & n.d. & n.d. & $\$ 493$ & $67 \%$ & 32.5 & 28,275 \\
\hline Cocineros & $\$ 226$ & $54 \%$ & $\$ 389$ & $53 \%$ & 32.1 & 643,284 \\
\hline Albañiles especializados, tabique, block y piedra & $\$ 506$ & $122 \%$ & $\$ 701$ & $95 \%$ & 31.3 & 47,263 \\
\hline Trabajadores lavanderías y tintorerías & $\$ 220$ & $53 \%$ & $\$ 391$ & $53 \%$ & 30.6 & 58,752 \\
\hline Hojalatería automotríz y similares & $\$ 403$ & $97 \%$ & $\$ 661$ & $89 \%$ & 30.5 & 49,715 \\
\hline Cortadores industriales & $\$ 315$ & $76 \%$ & $\$ 511$ & $69 \%$ & 29.9 & 24,219 \\
\hline
\end{tabular}

Fuente: Elaborada por la autora con datos de Employment and Earnings, enero de 2010. 
GRÁFICA 3

Medianas del ingreso hombres t. c. 1970-2008

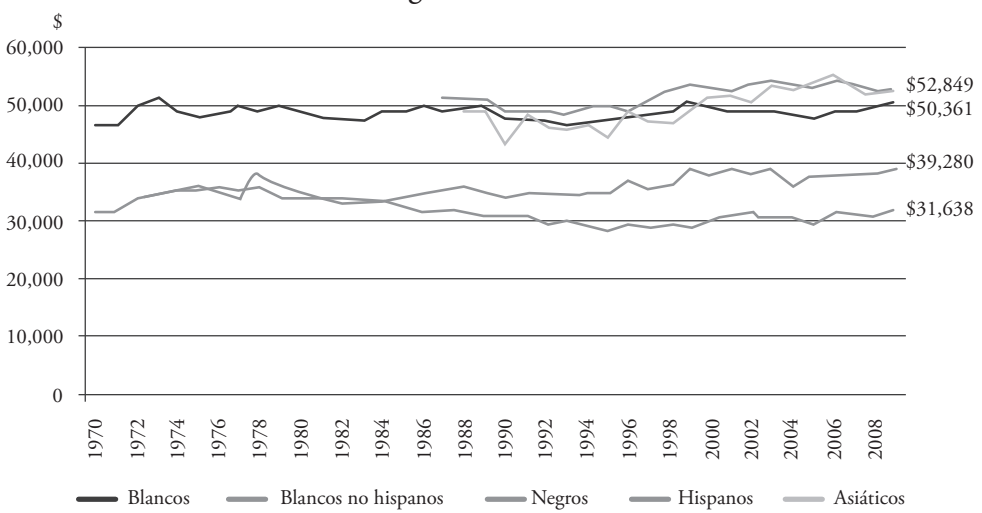

Fuente: Elaborada por la autora con datos de Current Population Survey, Historical Income Tables.

GRÁFICA 4

Medianas del ingreso mujeres t. c. 1970-2009

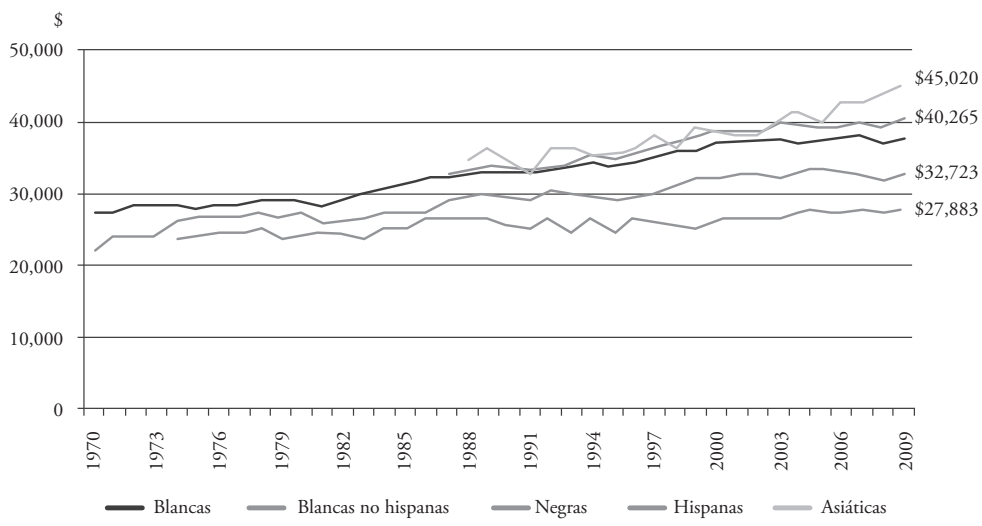

Fuente: Elaborada por la autora con datos de Current Population Survey, Historical Income Tables.

La mediana del ingreso de las mujeres latinas es marcadamente menor que la de las afroamericanas, quienes actualmente tienen un nivel bastante cercano a la de las blancas no hispanas. En el caso de las mujeres que tienen trabajos de tiempo completo a lo largo del año (ver gráfica 4), la mediana de las latinas ha sido la más baja consistentemente, desde que se registran datos al respecto, y la brecha tiende a crecer (us Census 
Bureau, 2010a). Cabe señalar que las tendencias para los trabajadores de tiempo completo, tanto hombres como mujeres, suelen tener ciertas divergencias con respecto al comportamiento de las medianas de ingresos de todos los que tienen algún empleo. Además, entre los trabajadores latinos, los mexicanos y las mexicanas, y algunos centroamericanos, suelen tener las medianas de ingresos más bajas, respectivamente. ${ }^{4}$

Por otra parte, aunque las medianas del ingreso de los hogares y de las familias latinos son un poco más altas que las de los afroamericanos, la brecha de ambos con respecto a las medianas de los hogares y las familias de los blancos no hispanos tienden a ensancharse ligeramente. Las diferencias no se deben a mejores remuneraciones para los latinos - ya hemos visto que tanto hombres como mujeres latinos tienden a ganar menos que los afroamericanos - sino al hecho de que hay un número mayor de trabajadores por familia u hogar. Pero al mismo tiempo suele haber un mayor número de dependientes. Muchas veces los hogares latinos incluyen miembros de su familia extendida - tíos, primos, sobrinos...- e inclusive personas que no son miembros de la familia, pero que probablemente provengan del mismo lugar de origen. El efecto es que el ingreso mayor se divide entre un mayor número de personas y, por lo tanto, desde 1985, el ingreso per capita de los latinos es menor que el de los afroamericanos. En 2009, la diferencia fue de 2,648 dólares anuales (15,063 y 17,711 dólares, respectivamente), y el ingreso per capita de los blancos no hispanos fue más de dos veces mayor (30,941 dólares) que el de los latinos (us Census Bureau, 2010a).

Es muy notable que, a nivel nacional, y no obstante los retrocesos sufridos en periodos de recesión, el índice de pobreza para los afroamericanos disminuyó a lo largo de los últimos 40 años del siglo Xx (de $55.1 \%$ en 1959 a un mínimo de $22.5 \%$ en 2000 ); a partir de entonces, se ha repuntado ligeramente, alcanzando el $25.9 \%$ en 2009. El caso de los latinos ha mostrado un comportamiento distinto (ver gráfica 5). Entre 1972 y 1994, la incidencia de pobreza para ellos fue más bien ascendente (pasó de $22.8 \%$ en 1972 a $30.7 \%$ en 1994), aunque ha

\footnotetext{
${ }^{4}$ Para un análisis más detallado de la estructura salarial y ocupacional de los latinos en Estados Unidos, ver Levine (2001, cap. 3; 2010:149-187).
} 
disminuido significativamente desde entonces. Alcanzó su nivel más bajo de $20.5 \%$ en 2006 y subió a $25.3 \%$ en 2009. Dicho indicador fue mayor para los latinos que para los afroamericanos durante cuatro años consecutivos (de 1994 a 1997). Dado el mayor crecimiento general de la población latina, mientras la participación de los afroamericanos en el conjunto de los pobres muestra por lo general una tendencia descendente de $31.1 \%$ del total en 1966 a $24.3 \%$ en 2009 (ver gráfica 6), la de los latinos creció marcadamente, de $10.3 \%$ en 1972 a $28.3 \%$ en 2009 (US Census Bureau, 2010b). En otras palabras, los latinos, que constituyen alrededor del $16 \%$ de la población estadounidense, son cerca del $30 \%$ de las personas que tienen ingresos por debajo del umbral de la pobreza. De continuarse las tendencias actuales, la población hispana en Estados Unidos será no solamente la minoría étnica o racial más numerosa - como fue constatado en el censo de 2000-, sino que pronto llegará a ser también la más depauperada. Además, la proporción de los migrantes recién llegados que vive cerca o debajo del umbral de la pobreza es considerablemente más alta.

GRÁFICA 5

Incidencia de pobreza 1959-2009

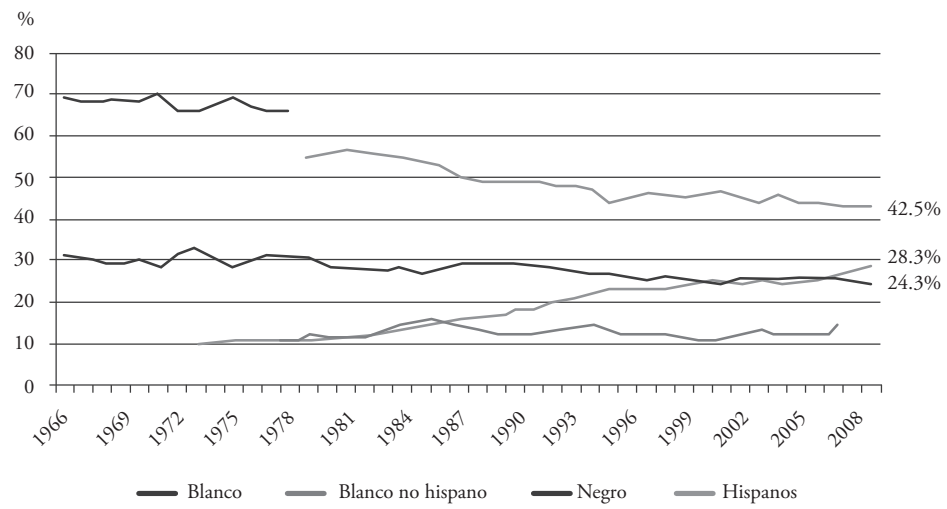

Fuente: Elaborada por la autora con datos de Current Population Survey, Historical Poverty Tables. 
GRÁFICA 6

Distribución de las personas pobres 1966-2009

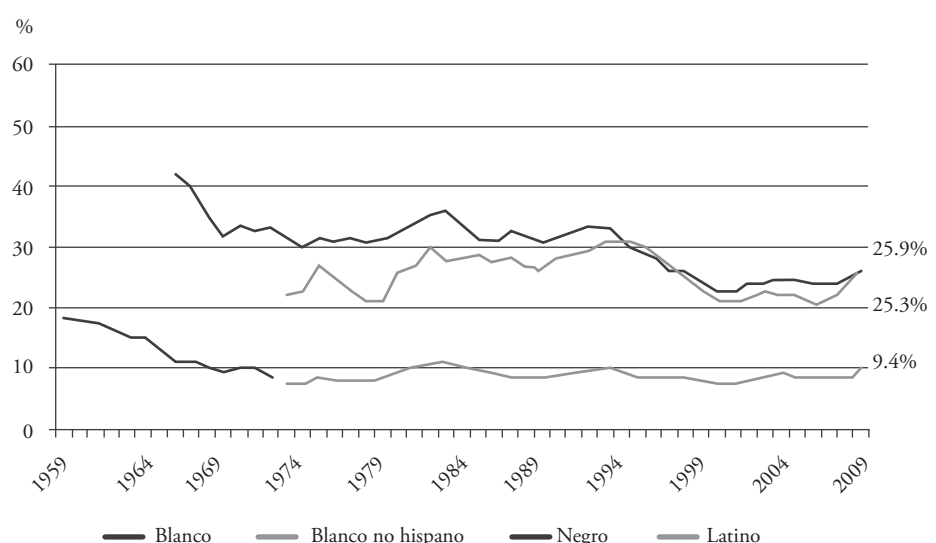

Fuente: Elaborada por la autora con datos de Current Population Survey, Historical Poverty Tables.

Las diferencias en ingresos y estatus socioeconómico pueden ser explicadas, en parte, por diferencias en escolaridad, sobre todo en décadas recientes con la correlación creciente entre niveles de escolaridad y niveles de ingresos que se observa en Estados Unidos. A pesar de esta creciente vinculación entre escolaridad e ingresos, y las cada vez más escasas perspectivas económicas para quienes no tienen estudios superiores - ni mucho menos para los que no terminaron siquiera high school-, persiste el problema de la deserción escolar, sobre todo para la población hispana. Por eso, acceder a la educación superior resulta doblemente difícil para la mayoría de los jóvenes latinos, hecho que a la vez limita sus opciones de empleo y, por consiguiente, las perspectivas de movilidad socioeconómica intergeneracional. Como se puede ver en las gráficas 7 y 8 , los latinos de origen mexicano son los rezagados de los rezagados en términos de escolaridad. 
GRÁFICA 7

Porcentaje sin certificado de high school 2009

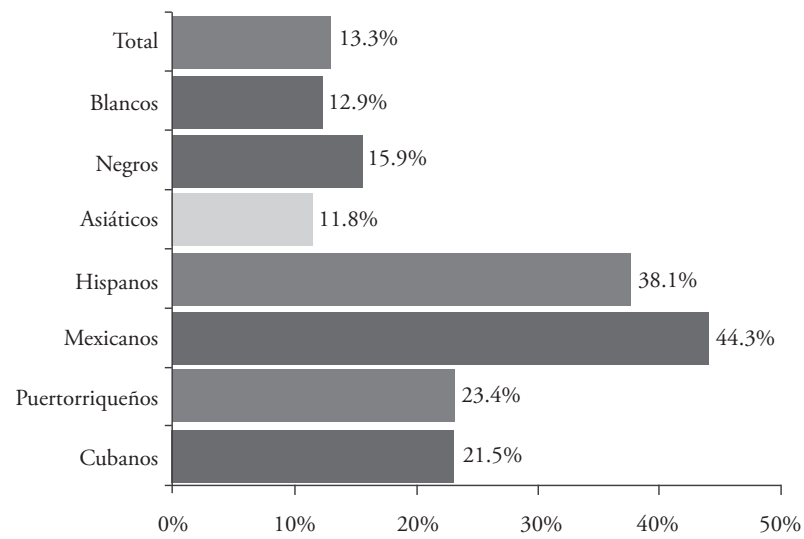

Fuente: Elaborada por la autora con datos del Statistical Abstract of the US 2011.

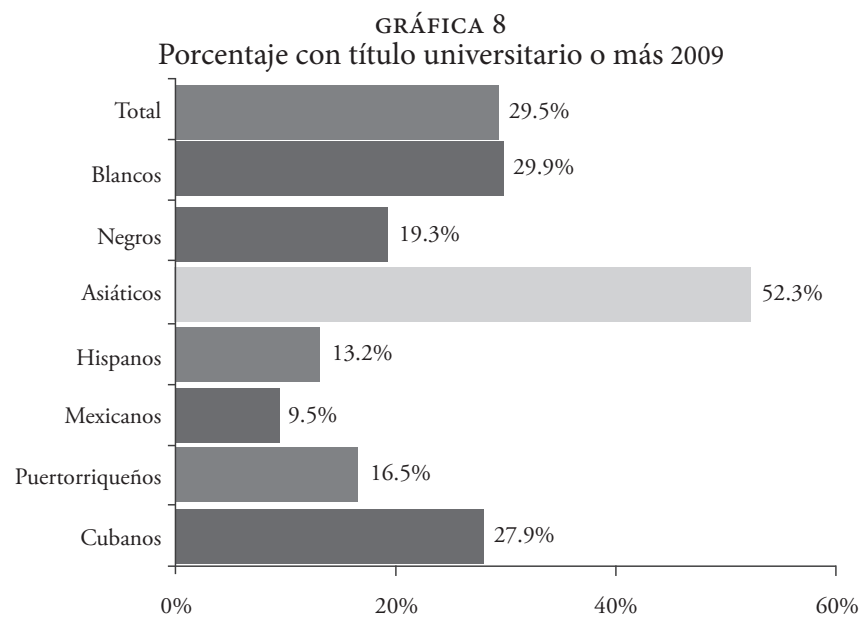

Fuente: Elaborada por la autora con datos del Statistical Abstract of the US 2011.

El alto porcentaje de mexicanos que no han concluido high school, o su equivalente en México que sería la preparatoria, se explica, en parte, porque México la educación obligatoria es hasta la secundaria. Inclusive hay un gran número de pueblos o ranchos que no cuentan con escuelas más allá de la primaria y donde tampoco existe el ciclo preescolar. $\mathrm{Al}$ concluir la secundaria, muchas familias consideran que sus hijos han 
llegado al final de la vida escolar y que están listos para trabajar; es el momento en que jóvenes provenientes de lugares con alto índice migratorio, suelen emprender su primer viaje al norte con el propósito de buscar empleo. Esto ayuda a explicar por qué en el contexto estadounidense los mexicanos tienen niveles de escolaridad tan bajos.

Dentro de cada uno de los grupos étnicos o raciales, los perfiles de escolaridad de hombres y mujeres muestran bastante similitud en la actualidad; sin embargo, persisten claras diferencias en los ingresos (ver gráfica 9). La diferencia en el promedio de ingresos que existe entre los hombres blancos y todos los demás es creciente, excepto en el caso de los hombres latinos con maestría, que son numérica y proporcionalmente pocos. Para cada nivel de escolaridad, los hombres afroamericanos y las mujeres latinas suelen ser un poco más castigados que sus contrapartes de los otros grupos.

GRÁFICA 9

Promedio de ingresos anuales según escolaridad 2008

$\$$

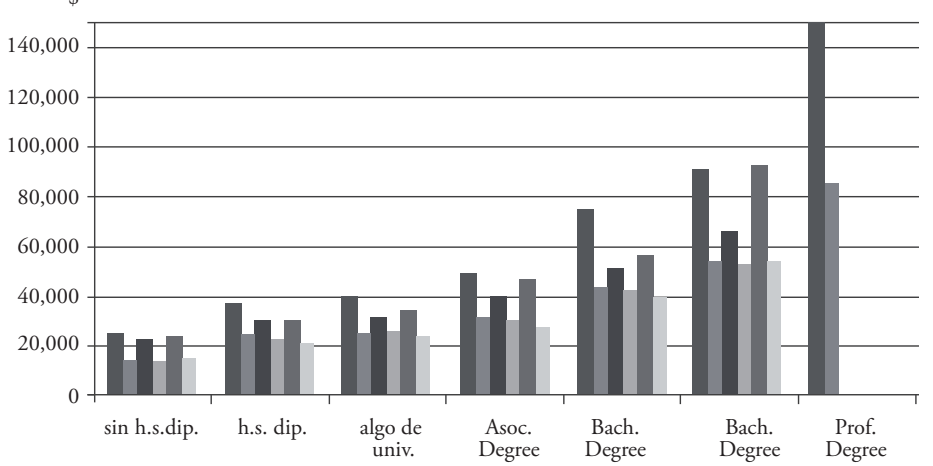

Hombres Blancos Mujeres Blancas

Hombres negros $\quad$ Mujeres negras

Hombres latinos

Mujeres latinas

Fuente: Elaborada por la autora con datos del Statistical Abstract of the US 2011. 


\section{CONSIDERACIONES FINALES}

El mercado laboral estadounidense ha experimentado cambios radicales en las últimas décadas como resultado de las respuestas que este país ha dado para enfrentar la globalización y la creciente competencia internacional. Los empleos en casi todos los niveles se han vuelto más inestables, y muchos se han vuelto francamente precarios. Para bajar costos laborales, muchas empresas han comprimido los salarios, mientras la innovación tecnológica hace posible eliminar miles de puestos de trabajo. Pero, a la vez que desaparecen empleos en la manufactura tradicional, surgen otros en un sinnúmero de nuevos servicios. Los patrones de oferta y demanda de mano de obra han cambiado marcadamente. Aun cuando muchos de los nuevos empleos creados en Estados Unidos son despreciados por quienes han sido desplazados de puestos manufactureros, que pagaban bastante bien, hasta 2007 había una afluencia constante y creciente de inmigrantes mexicanos dispuestos a aceptar casi cualquier trabajo que les proporcionara un ingreso en dólares.

La situación de crisis que se manifestó a finales de 2007 plantea condiciones nuevas y cambiantes que no se pueden analizar adecuadamente hasta que se vean con claridad la duración, la profundidad y los impactos de esta recesión. Por el momento, se ha frenado el ritmo de las migraciones internacionales, en particular de la migración de mexicanos a Estados Unidos. Es de esperarse que, con la eventual reactivación de las principales economías del mundo, se reactivarán los flujos migratorios, aunque tal vez con algunas modificaciones, todavía imprevisibles, en sus patrones anteriores. En el caso de Estados Unidos, por el perfil demográfico del país, que se caracteriza por el rápido envejecimiento de la población, y una demanda creciente, en tiempos de expansión económica, para varios tipos de servicios, y otras actividades como la construcción, que no puede ser satisfecha más que in situ, es razonable suponer que la reactivación económica será acompañada, hasta cierto punto, por una renovada demanda de mano de obra inmigrante en ambos extremos del espectro laboral. Por lo tanto, es razonable suponer que se reanudará, en buena medida, el flujo migratorio de mexicanos a Estados Unidos, y su incorporación al mercado laboral, en términos más o menos similares a los que se describen en este trabajo. 
En cuanto a la interrogante planteada por Rifkin sobre el fin del trabajo, parece bastante evidente que muchas formas tradicionales de trabajo están desapareciendo, al tiempo que están surgiendo nuevas formas, muchas de ellas derivadas de la revolución informática, y muchas otras vinculadas con la expansión y liberalización de las relaciones mercantiles. El mercado invade hoy día prácticamente todos los ámbitos de la vida. En un libro posterior, La era del acceso, Rifkin (2001) responde a su propio cuestionamiento sobre el fin del trabajo aseverando que, en general, habrá nuevas oportunidades de empleo, pero se darán en el ámbito del comercio y los servicios. Mientras, cada vez más aspectos de la vida humana se convierten en experiencias por las que se paga, es decir, se comercializan, por lo que millones de personas estarán empleadas para proveer y comercializar los deseos y las necesidades de otros. Estamos experimentando la mercantilización de cada vez más aspectos de la vida humana. Estas actividades se están convirtiendo en transacciones mercantiles que sustituyen las relaciones sociales tradicionales, y ya estamos presenciando los trastornos sociales que acompañan está transformación. No se trata, por tanto, del fin del trabajo, sino de un cambio radical del trabajo y de las propias relaciones humanas.

\section{BIBLIOGRAFÍA}

Durand, Jorge y Douglas S. Massey (1982-2011), The Mexican Migration Project, http://mmp.opr.princeton.edu/

Levine, Elaine (2001), Los Nuevos Pobres de Estados Unidos: Los Hispanos. México, Miguel Ángel Porrúa/IIE/Cisan/unam.

(2006), «Hijos de migrantes mexicanos en las escuelas de Estados Unidos» en Sociológica, núm. 60, enero-abril de 2006.

(2010), «Los hispanos/latinos en Estados Unidos» en Migración México-Estados Unidos: Textos introductorios, José Luis Sánchez Gavi y Adriana Sletza Ortega Ramírez (coord.), BUAP, Puebla, pp. 149-187.

Passel, Jeffrey S. (2005), Unauthorized Migrants: Numbers and Characteristics, Washington, Pew Hispanic Center.

(2006), The Size and Characteristics of the Unauthorized Migrant Population in the US, Washington, Pew Hispanic Center. 
Levine, Elaine y Cohn D’Vera (2010), Unauthorized Immigrant Population: National and State Trends, 2010. Washington, Pew Hispanic Center. Reich, Robert (1992), The Work of Nations, New York, Vintage Books. Rifkin, Jeremy (1996), The End of Work, New York, G.P. Putnam's Sons. (2001), La Era del Acceso, New York, Pinguin Putnam.

Sum, Andrew, Neeta Fogg, Paul Harrington et al. (2002), «Immigrant Workers and the Great American Job Machine: The Contributions of New Foreign Immigration to National and Regional Labor Force Growth in the 1990s», trabajo preparado para el National Business Roundtable, Washington, agosto de 2002.

Thomas-Breitfeld, Sean (2003), "The Latino Workforce», Statistical Brief, núm. 3. Washington, National Council of La Raza.

UChitelle, Louis y David Leonhardt (2006), «Men not working, and not wanting just any job», en The New York Times, 31 de julio de 2006, http:// wwwnytimes.com/2006/07/31/business/31men.html

us Census Bureau. (1983), Statistical Abstract of the United States. 1984, Washington, United States Government Printing Office.

(2001a), UsA Statistics in Brief-1990 and 2000 Census Race and Hispanic Data Internet, http://www.census.gov/statab/www/part1a.html consultado el 25 de marzo de 2001.

(2001b), The Hispanic Population, Census 2000 Brief. с2кв/01-3, mayo.

(2010a), March Current Population Survey, Historical Income Tables http://www.census.gov/hhes/www/income/data/historical/families/ index.htmlhttp://www.census.gov/hhes/www/income/data/historical/household/H05_2009.xls, http://www.census.gov/hhes/www/income/data/historical/people/index.html, consultado en febrero de 2011 (2010b), March Current Population Survey, Historical Poverty Tables, table 2, http://www.census.gov/hhes/www/poverty/data/historical/ hstpov2.xls, consultado en febrero de 2011.

(2011), The 2011 Statistical Abstract. http://www.census.gov/compendia/statab/, consultado en febrero de 2011.

USDOL (1984), Employment and Earnings, vol. 31, núm. 1, Washington, UsGPO. (1991) Employment and Earnings, vol. 38, núm. 1, Washington, UsGPO. (2004) Employment and Earnings, vol. 51, núm. 1, Washington, UsGPO. (2005) Employment and Earnings, vol. 52, núm. 1, Washington, UsGPO. (2006) Employment and Earnings, vol. 53, núm. 1, Washington, USGPO. 
(2009a), Employment and Earnings, vol. 56, núm. 1, Washington. Bureau of Labor Statistics, http://www.bls.gov/opub/ee/empearn200901.pdf (2009b), «Economic News Release, Employment projections 20082010 Summary», Washington, Bureau of Labor Statistics, http://www. bls.gov/news.release/ecopro.nr0.htm, http://www.bls.gov/news.release/ ecopro.t06.htm, http://www.bls.gov/news.release/ecopro.t07.htm, consultado en febrero de 2011.

(2010), Employment and Earnings, vol. 57, núm. 1,Washington, D. C., Bureau of Labor Statistics, http://www.bls.gov/opub/ee/empearn201001.pdf (2011), Employment and Earnings, vol. 58, núm. 1,Washington, D. C., Bureau of Labor Statistics, http://www.bls.gov/opub/ee/empearn201101.pdf WIAL, Howard y Alec Friedhoff (2006), «Bearing the Brunt: Manufacturing Job Loss in the Great Lakes Region, 1995-2005», Washington, The Brookings Institution. 